\title{
Commensurate to incommensurate magnetic phase transition in honeycomb-lattice pyrovanadate $\mathrm{Mn}_{2} \mathbf{V}_{\mathbf{2}} \mathrm{O}_{7}$
}

\author{
J. Sannigrahi, D. T. Adroja, R. Perry, M. J. Gutmann, \\ V. Petricek and D. Khalyavin
}

\section{Published version information}

Citation: J Sannigrahi et al. "Commensurate to incommensurate magnetic phase transition in honeycomb-lattice pyrovanadate $\mathrm{Mn}_{2} \mathrm{~V}_{2} \mathrm{O}_{7}$." Physical Review Materials, vol. 3, no. 11 (2019): 113401.

DOI: $10.1103 /$ PhysRevMaterials.3.113401

This version is made available in accordance with publisher policies. Please cite only the published version using the reference above. This is the citation assigned by the publisher at the time of issuing the APV. Please check the publisher's website for any updates. 


\title{
Commensurate to incommensurate magnetic phase transition in honeycomb-lattice pyrovanadate $\mathrm{Mn}_{2} \mathrm{~V}_{2} \mathrm{O}_{7}$
}

\author{
J. Sannigrahi,,${ }^{1, *}$ D. T. Adroja $\odot,{ }^{1,2, \dagger}$ R. Perry,${ }^{1,4}$ M. J. Gutmann, ${ }^{1}$ V. Petricek,${ }^{3}$ and D. Khalyavin ${ }^{1}$ \\ ${ }^{1}$ ISIS Facility, Rutherford Appleton Laboratory, STFC, Chilton, Didcot OX11 0QX, United Kingdom \\ ${ }^{2}$ Highly Correlated Matter Research Group, Physics Department, University of Johannesburg, P.O. Box 524, \\ Auckland Park 2006, South Africa \\ ${ }^{3}$ Institute of Physics, Czech Academy of Sciences, 18040 Prague, Czech Republic \\ ${ }^{4}$ Centre for Materials Discovery and London Centre for Nanotechnology, University College London, London, United Kingdom
}

(Received 21 May 2019; revised manuscript received 27 September 2019; published 18 November 2019)

\begin{abstract}
We have synthesized a single-crystalline sample of $\mathrm{Mn}_{2} \mathrm{~V}_{2} \mathrm{O}_{7}$ using the floating zone technique, and we investigated the ground state using magnetic susceptibility, heat capacity, and neutron diffraction. Our magnetic susceptibility and heat capacity reveal two successive magnetic transitions at $T_{N 1}=17 \mathrm{~K}$ and $T_{N 2}=11.8 \mathrm{~K}$ indicating two distinct magnetically ordered phases. The single-crystal neutron diffraction study shows that in the temperature $(T)$ range $11.8 \leqslant T \leqslant 17 \mathrm{~K}$ the magnetic structure is commensurate with propagation vector $k_{1}=(0,0.5,0)$, while upon lowering temperature below $T_{N 2}=11.8 \mathrm{~K}$ an incommensurate magnetic order emerges with $k_{2}=(0.38,0.48,0.5)$ and the magnetic structure can be represented by cycloidal modulation of the Mn spin in the ac plane. We discuss the role of magnetic exchange interactions and spin-orbital coupling on the stability of the observed magnetic phase transitions.
\end{abstract}

DOI: 10.1103/PhysRevMaterials.3.113401

\section{INTRODUCTION}

Bulk crystals are inherently three-dimensional; however, they may consist of magnetic ions whose spins interact only along one or two certain crystallographic directions. Such compounds are known as low-dimensional magnets, which have attracted considerable attention in solid-state chemistry and physics due to their nontypical behavior, which mainly involves electrical and magnetic properties. Two-dimensional honeycomb-lattice systems are one of the interesting lowdimensional magnetic systems that have been investigated intensively because of novel ground states induced by frustration and quantum fluctuations [1-4]. Magnetic order can be obtained on a regular two-dimensional lattice assuming antiferromagnetic interactions between adjacent spins [5], or on a bilayer lattice with frustrating interlayer interactions [6]. In recent times, these honeycomb-lattice systems have been investigated extensively both theoretically and experimentally as potential candidates for a Kitaev quantum spin liquid state, e.g., $\mathrm{Na}_{2} \mathrm{IrO}_{3}$ and $\alpha-\mathrm{RuCl}_{3}$ [4,7-9]. Moreover, superconductivity has also been observed in pnictide SrPtAs with a honeycomb lattice [10].

The title compound $\mathrm{Mn}_{2} \mathrm{~V}_{2} \mathrm{O}_{7}$ is a member of the family of transition-metal-vanadium oxides $M_{2} \mathrm{~V}_{2} \mathrm{O}_{7}(M=$ $\mathrm{Cu}, \mathrm{Ni}, \mathrm{Co}, \mathrm{Mn}$ ), which have attracted much interest due to their rich structural features and magnetic properties [11-20]. $\mathrm{Mn}_{2} \mathrm{~V}_{2} \mathrm{O}_{7}$ is composed of magnetic $\mathrm{Mn}^{2+}\left(3 d^{5}, S=5 / 2\right)$ and nonmagnetic $\mathrm{V}^{5+}\left(3 d^{0}, S=0\right)$ ions, and it was reported to possess a quasi-two-dimensional distorted honeycomb lat-

\footnotetext{
*jhuma.iacs@gmail.com

†devashibhai.adroja@stfc.ac.uk
}

tice [21,22]. It exhibits two different structural phases, namely $\beta$-phase (above $\approx 310 \mathrm{~K}$ ) with monoclinic $(C 2 / m)$ symmetry and $\alpha$-phase (below $\approx 284 \mathrm{~K}$ ) with triclinic $(P \overline{1})$ symmetry. As temperature decreases, the high-temperature monoclinic symmetry of $\mathrm{Mn}_{2} \mathrm{~V}_{2} \mathrm{O}_{7}$ reduces to triclinic symmetry, and this structural transition is completely reversible with reasonable thermal hysteresis, in line with the martensitic-like first-order nature of this structural transition involving lattice distortion but without atomic exchange $[18,22]$. Depending on the synthesis procedure, these transition temperatures vary because of the change in $\mathrm{O}_{2}$ pressure and cooling rate. The honeycomb networks of Mn-atoms in the $\beta$-phase are parallel to the (001) plane, and those in the $\alpha$-phase are parallel to the (1-10) plane. In addition, the sample undergoes a paramagnetic to antiferromagnetic transition below around $17 \mathrm{~K}$. It is noted that such properties of $\mathrm{Mn}_{2} \mathrm{~V}_{2} \mathrm{O}_{7}$ were mainly based on macroscopic characterization methods on polycrystalline as well as fluxgrown single-crystalline samples. But a detailed microscopic model of the magnetism has not yet been developed.

There have only been a few reports on the single-crystal growth of $\mathrm{Mn}_{2} \mathrm{~V}_{2} \mathrm{O}_{7}$ where only the flux-growth technique has been followed $[23,24]$. The crystals obtained are small, and moreover the grown crystals may incorporate traces of the molten flux or the crucible materials, which is highly undesirable. Keeping this view in mind, we decided to grow $\mathrm{Mn}_{2} \mathrm{~V}_{2} \mathrm{O}_{7}$ crystals using the traveling solvent floating zone (TSFZ) method associated with an optical image furnace. $\mathrm{Mn}_{2} \mathrm{~V}_{2} \mathrm{O}_{7}$ melts congruently upon heating at (1080 \pm $3)^{\circ} \mathrm{C}$ [25]. To understand the structural and magnetic properties of the ground state of $\mathrm{Mn}_{2} \mathrm{~V}_{2} \mathrm{O}_{7}$ at the macroscopic as well as microscopic level, we carried out $\mathrm{x}$-ray diffraction (XRD), magnetization, and heat capacity measurement along with the single-crystal neutron diffraction study. 


\section{EXPERIMENTAL DETAILS}

The single-crystalline sample of $\mathrm{Mn}_{2} \mathrm{~V}_{2} \mathrm{O}_{7}$ has been grown in a four-mirror optical floating zone furnace from Crystal System Corporation, Japan. The furnace consists of four halogen lamps. Every lamp takes the focus position of an ellipsoidal reflector, whereas the second focus of the reflectors coincides at a point inside the growth chamber on the vertical axis of the furnace. A molten zone between the polycrystalline feed rod and the seed rod is formed at this common focal point where the energy flux of the lamps converges after reflecting from the reflectors. The feed rod is suspended from the upper shaft with a nickel wire while the seed rod is clamped to the lower shaft using an alumina holder. A polycrystalline sample of $\mathrm{Mn}_{2} \mathrm{~V}_{2} \mathrm{O}_{7}$ was prepared by the standard solid-state reaction route in air. Highly pure $\mathrm{MnO}_{2}$ and $\mathrm{V}_{2} \mathrm{O}_{5}$ were mixed thoroughly in a stoichiometric ratio in an agate mortar. The mixture was pressed into pellets and sintered at $600^{\circ} \mathrm{C}$ for one week with several intermediate grindings. The phase analysis of the final product was performed using powder X-ray diffraction (Miniflex). The single-phase powder of $\mathrm{Mn}_{2} \mathrm{~V}_{2} \mathrm{O}_{7}$ was filled in a rubber tube and cold pressed under 700 bar isostatic pressure to obtain uniform rods. The rods formed in this process are $5 \mathrm{~mm}$ in diameter and 70-90 $\mathrm{mm}$ in length. Then the rods were sintered in the platinum boat at a temperature of $900{ }^{\circ} \mathrm{C}$ overnight to obtain very dense and homogeneous rods. This process ensures the homogeneous melting of the feed rod during the growth process. A part about $20 \mathrm{~mm}$ long was cut from the rods and used as the seed rod.

Three different growth experiments were performed with different oxygen pressure and growth speed employed: (i) $60 \% \mathrm{O}_{2}+40 \%$ Ar and growth speed $5 \mathrm{~mm} / \mathrm{h}$, (ii) $100 \% \mathrm{O}_{2}$ flow $\left(P=7\right.$ bar) and growth speed $5 \mathrm{~mm} / \mathrm{h}$, and (iii) $100 \% \mathrm{O}_{2}$ flow $(P=7$ bar $)$ and growth speed $1 \mathrm{~mm} / \mathrm{h}$. The growth process is initiated by fusing the bottom end of the feed rod inside the optical furnace. In our case, $300 \mathrm{~W}$ lamps have been installed. The upper and lower shafts of the furnace were rotated in opposite directions with a rotation speed $30 \mathrm{rpm}$ to improve the temperature and compositional homogeneity of the float zone. In the first two attempts, the upper shaft was translated at a speed of $1 \mathrm{~mm} / \mathrm{h}$, and in the third attempt it was translated at a speed of $0.5 \mathrm{~mm} / \mathrm{h}$ in order to control the steady state of the floating zone. In the first attempt, the molten zone was not fully stable, and the formation of bubbles was observed near the upper edge of the zone possibly due to the reduction of $\mathrm{Mn}$ ions. Then we decided to use $100 \% \mathrm{O}_{2}$ pressure to prevent the reduction of $\mathrm{Mn}^{2+}$, and with this environment we got a significantly stable zone throughout the growth process as shown in the right inset of Fig. 1(a).

The grown crystals were characterized using powder x-ray diffraction (XRD) of crushed crystal (Bruker D8 Advance), Laue X-ray diffraction and single-crystal neutron diffraction in the SXD diffractometer, ISIS neutron and muon facility. The magnetization of the crystals was measured by applying an external field along the three different crystallographic directions using a vibrating sample magnetometer (SQUID VSM, Quantum Design) in the temperature range $T=2-320 \mathrm{~K}$ and applied magnetic field $(H)$ ranging from -70 to $+70 \mathrm{kOe}$. Heat capacity has been measured in Quantum Design PPMS. The $\mathrm{x}$-ray diffraction (XRD) pattern of crushed crystal
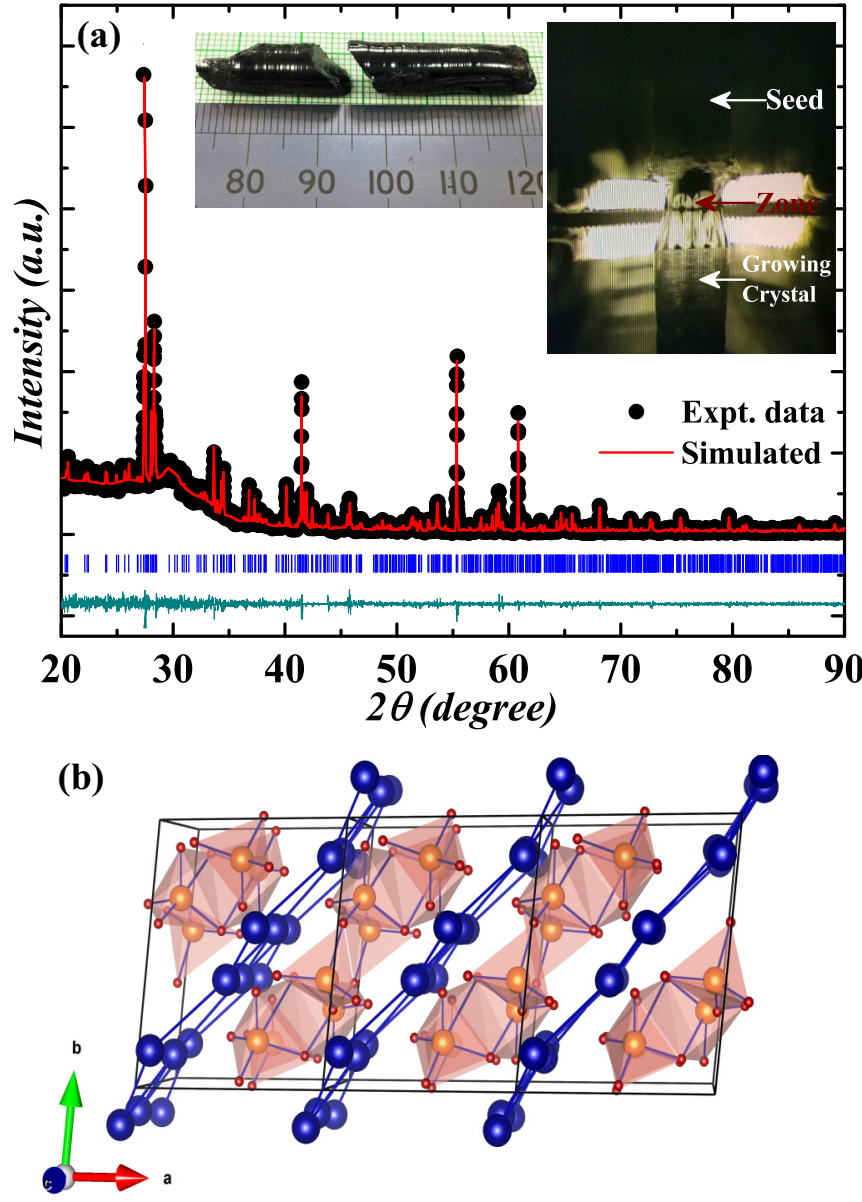

FIG. 1. (a) The main panel shows the XRD pattern of a crushed single crystal measured at $T=100 \mathrm{~K}$. The black scattered points and red solid line represent, respectively, the experimental data and the simulated curve obtained from Rietveld refinement. Blue ticks indicate the Bragg positions for triclinic $P \overline{1}$ structure, and the cyan line shows the difference between an experimental and a calculated pattern. The right inset shows the stable solvent zone formation during growing, while the left inset depicts the picture of the asgrown crystal. Part (b) depicts the perspective view of the crystal structure of $\mathrm{Mn}_{2} \mathrm{~V}_{2} \mathrm{O}_{7}$ at $100 \mathrm{~K}$ where blue, yellow, and red spheres represent $\mathrm{Mn}, \mathrm{V}$, and $\mathrm{O}$ atoms, respectively.

measured at $100 \mathrm{~K}$ is shown in the main panel of Fig. 1(a). Rietveld refinement on powder XRD data was performed using the FULLPROF software package, and the data are wellfitted using triclinic structure with the $P \overline{1}$ space group. The details of the refinement are provided in the Supplemental Material [26]. The crystal structure [shown in Fig. 1(b)] is consistent with the literature.

\section{MAGNETIZATION AND HEAT CAPACITY}

The main panel of Fig. 2(a) shows the temperature dependence of magnetic susceptibility $(\chi)$ from 2 to $110 \mathrm{~K}$, which was measured in the zero-field-cooled heating protocol under an applied magnetic field of $H=100$ Oe along the crystallographic directions of $\alpha-\mathrm{Mn}_{2} \mathrm{~V}_{2} \mathrm{O}_{7}$ parallel to [110], [1-10], and [001]. A clear signature of an antiferromagnetic (AFM) transition is observed at $T_{N 1} \approx 17 \mathrm{~K}$, which matches quite well with the previously reported data [21,22,27]. 

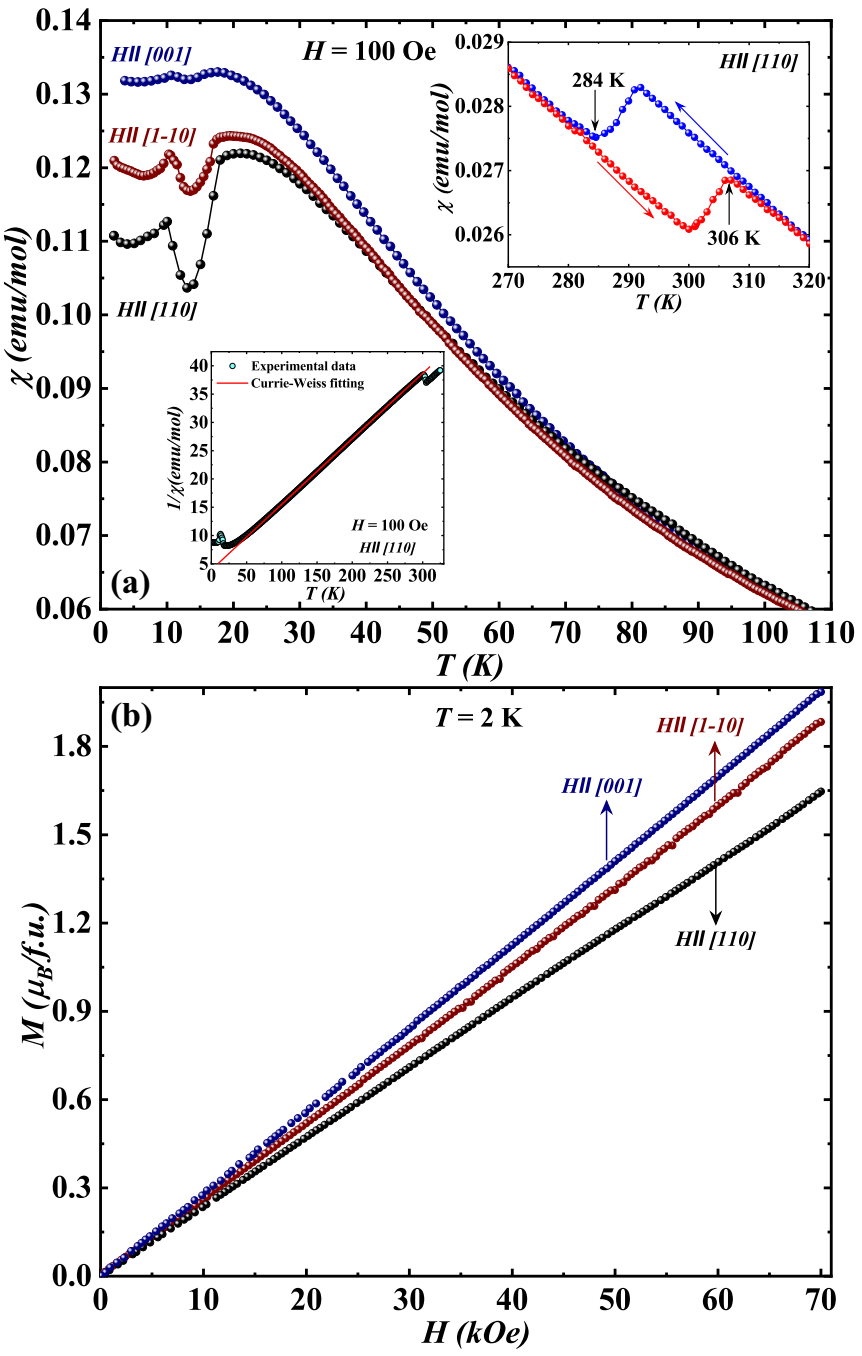

FIG. 2. (a) The main panel shows the temperature-dependent magnetization data in the zero-field-cooled heating protocol where magnetic field applied along three crystallographic directions: $H \|$ [110], $H \|$ [1-10], and $H \|$ [001]. The upper inset depicts the thermal hysteresis observed between 284 and $306 \mathrm{~K}$ on field cooling and field-cooled heating measurements, whereas the lower inset represents the Curie-Weiss fitting in the paramagnetic region. (b) $M$ vs $H$ curves are displayed measured at $T=2 \mathrm{~K}$ along three different crystallographic directions.

Additionally, we found another anomaly at around $T_{N 2}=$ $11.8 \mathrm{~K}$ in all three crystallographic directions. Although $T_{N 1}$ and $T_{N 2}$ are present at the same temperatures, different histories are clearly observed below $17 \mathrm{~K}$ depending on the direction of the applied magnetic field. This indicates the presence of magnetic anisotropy in this system. The upper inset of Fig. 2(a) shows the thermal hysteresis within 284 and $306 \mathrm{~K}$ between field-cooling and field-cooled-heating protocols of measurement. The existence of hysteresis suggests that this reversible structural transition is a first-order martensitic-like transition where a thermoelastic solid-solid phase transition occurs involving lattice distortion but without atomic exchange. The $1 / \chi(T)$ curve follows the Curie-Weiss law above $50 \mathrm{~K}$ in the $\alpha$-phase of the compound as shown in the lower inset of Fig. 2(a). We obtained Curie-Weiss $T$, $\theta_{C}=-34 \mathrm{~K}$ indicating the predominant AFM interaction,

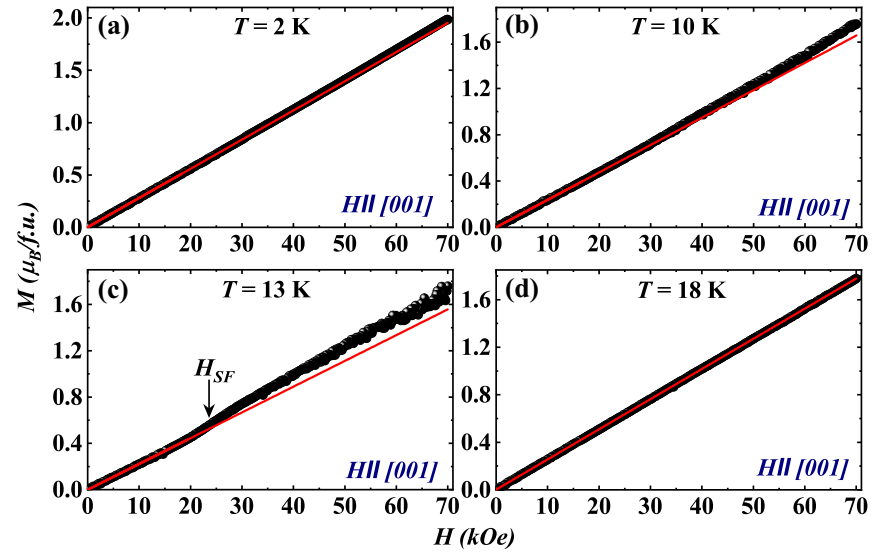

FIG. 3. $M$ vs $H$ curves are displayed in (a)-(d) measured correspondingly at $T=2,10,13$, and $18 \mathrm{~K}$ along the $H \|$ [001] direction. The red straight line is the guide to follow the linearity of the curve.

and an effective paramagnetic moment, $\mu_{\mathrm{eff}}=5.86 \mu_{B} / \mathrm{Mn}^{2+}$, which is nearer to the spin-only value $5.92 \mu_{B}$ of $\mathrm{Mn}^{2+}$. Figure 2(b) shows the isothermal magnetization measured at $T=2 \mathrm{~K}$ under an applied magnetic field up to $70 \mathrm{kOe}$, where $H \|$ [110], $H \|$ [1-10], and $H \|$ [001]. The linear nature of the $M$ versus $H$ curves in all three directions indicates again the presence of predominant AFM interaction in the studied compound. From the magnetic susceptibility and magnetization isotherm measurements, it is clear that the $c$-axis is an easy magnetization axis with weak anisotropy. Careful observation shows some deviation from linearity in the $M$ versus $H$ curve at low temperature measured along $H \|$ [001]. A very slight nonlinearity is observed at $T=2$ and $10 \mathrm{~K}$ as shown in Figs. 3(a) and 3(b). But at $T=13 \mathrm{~K}$, a clear jump is observed at $H_{\mathrm{SF}} \approx 23 \mathrm{kOe}$ as shown in Fig. 3(c) and is the signature of the spin-flop transition, which is also mentioned in the literature $[20,27]$. This observation is clearly evidence of magnetic anisotropy.

The heat capacity $\left(C_{p}\right)$ as a function of temperature measured at zero applied magnetic field is depicted in Fig. 4(a). At high temperature, $C_{p}$ is completely dominated by phonon excitations. At low temperature, two $\lambda$-type anomalies are observed at $T_{N 1} \approx 17 \mathrm{~K}$ and $T_{N 2} \approx 11.8 \mathrm{~K}$, indicating two magnetic phase transitions consistent with the magnetization measurement. To gain more information about magnetic ordering, $C_{p}(T)$ has been measured in a different applied magnetic field from 0 Oe to $90 \mathrm{kOe}$, which is shown in Figs. 4(b) and 4(c). With increasing $H, T_{N 1}$ shifts toward lower temperature suggesting the antiferromagnetic nature of this transition. In addition to this, the intensities of the peaks are decreasing with increasing magnetic field because of the redistribution of magnetic entropy. On the other hand, the peak at $T_{N 2}$ shifts slightly toward higher temperature from $H=0$ Oe to $30 \mathrm{kOe}$ up to a maximum $T_{N 2} \approx 12.8 \mathrm{~K}$ and decreases more clearly with a further increase of $H$. This peak also becomes broader with increasing $H$ and finally is completely suppressed above $50 \mathrm{kOe}$.

\section{SINGLE-CRYSTAL NEUTRON DIFFRACTION}

A single-crystal neutron diffraction study was carried out on the SXD diffractometer at the ISIS Neutron and Muon 

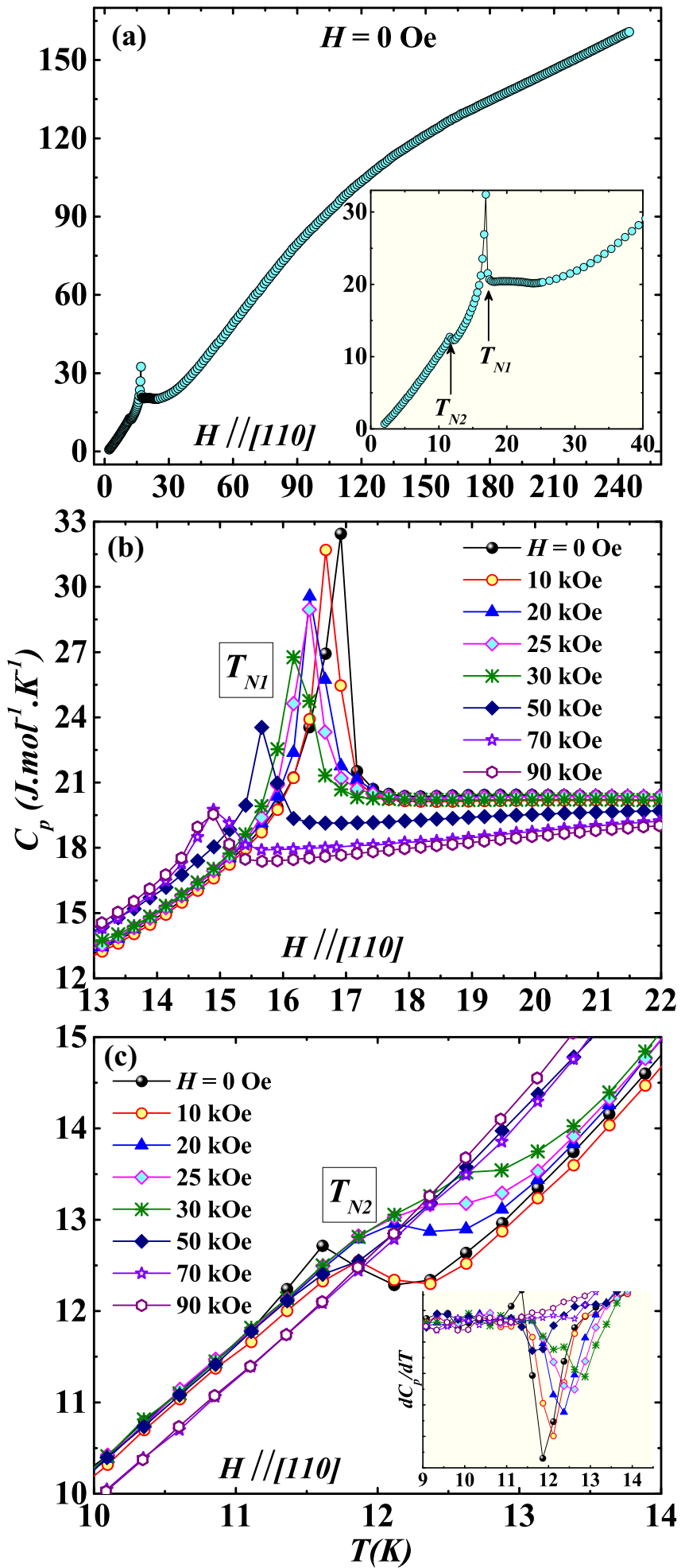

FIG. 4. (a) The main panel shows the temperature-dependent heat capacity measured at zero applied magnetic field while the inset shows the enlarged view of the low- $T$ regime indicating the two transitions at $T_{N 1}$ and $T_{N 2}$. The magnetic-field dependence of $T_{N 1}$ and $T_{N 2}$ is depicted in (b) and (c), respectively. $d C_{p} / d T$ vs $T$ is plotted in the inset of (c) to show clearly the change in $T_{N 2}$ with $H$.

facility, which utilizes the time-of-flight Laue technique [28]. A $\mathrm{Mn}_{2} \mathrm{~V}_{2} \mathrm{O}_{7}$ single crystal of approximate dimensions $2 \times 1 \times 0.5 \mathrm{~mm}^{3}(m \approx 50 \mathrm{mg})$ was mounted at the end of
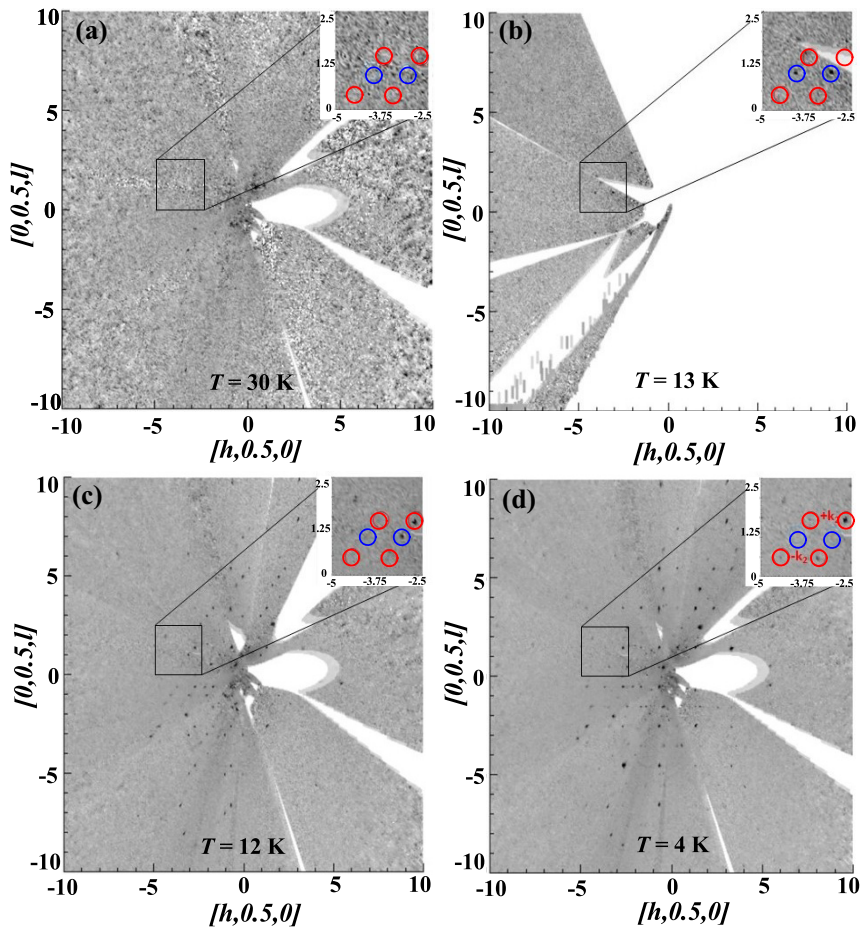

FIG. 5. 2D intensity maps of the reciprocal $(h, 0.5, l)$ planes at $30,13,12$, and $4 \mathrm{~K}$ are depicted in (a), (b), (c), and (d), respectively. The magnetic Bragg peaks for the commensurate propagation vector $k_{1}=(0,0.5,0)$ are shown by a blue circle, while the magnetic peaks for the incommensurate propagation vector $k_{2}=(0.38,0.48,0.5)$ are indicated by a red circle. The integration range \pm 0.025 was used for $k$. The insets depict the enlarged view of the particular area to show the magnetic Bragg peaks arising from $k_{1}$ and $k_{2}$.

an aluminum pin using adhesive $\mathrm{Al}$ tape. For a reliable determination of the propagation vector, single-crystal neutron diffraction data were collected at different temperatures ranging from 4 to $30 \mathrm{~K}$ with special attention at temperatures $T=4,12,13$, and $30 \mathrm{~K}$ using a closed-cycle He refrigerator. Five exposures in different crystal orientations with respect to the incident neutron beam were collected for $1 \mathrm{~h}$ each. The data were processed using locally available SXD2001 software [29]. Structural refinements of the neutron data were performed with the JANA2006 [30] software using standard scattering length densities and the $\mathrm{Mn}^{2+}$ form factor. The neutron intensity in a part of the reciprocal $(h, 1 / 2, l)$ scattering plane in $\alpha-\mathrm{Mn}_{2} \mathrm{~V}_{2} \mathrm{O}_{7}$ is shown as two-dimensional intensity maps in Fig. 5 for $T=30,13,12$, and $4 \mathrm{~K}$. To confirm the nuclear structure, a refinement of the data set collected at $30 \mathrm{~K}$ (well above the peak in the magnetic susceptibility and heat capacity) has been performed that confirms the triclinic structure with the $P \overline{1}$ space group. The crystal structure parameters are consistent with the literature [23,27]. In the intermediate phase (in between $T_{N 2}$ and $T_{N 1}$ ) we observed a set of magnetic superstructure peaks corresponding to the commensurate propagation vector $k_{1}=(0,0.5,0)$, which are marked by a blue circle in Fig. 5(b) at $T=13 \mathrm{~K}$. But in the ground state, below $T_{N 2}=11.8 \mathrm{~K}$, this commensurate phase disappears and a different set of magnetic superstructure peaks arises as shown in Fig. 5(d) by a red circle. Taking the positions of this new set of magnetic peaks, it is evident that the 


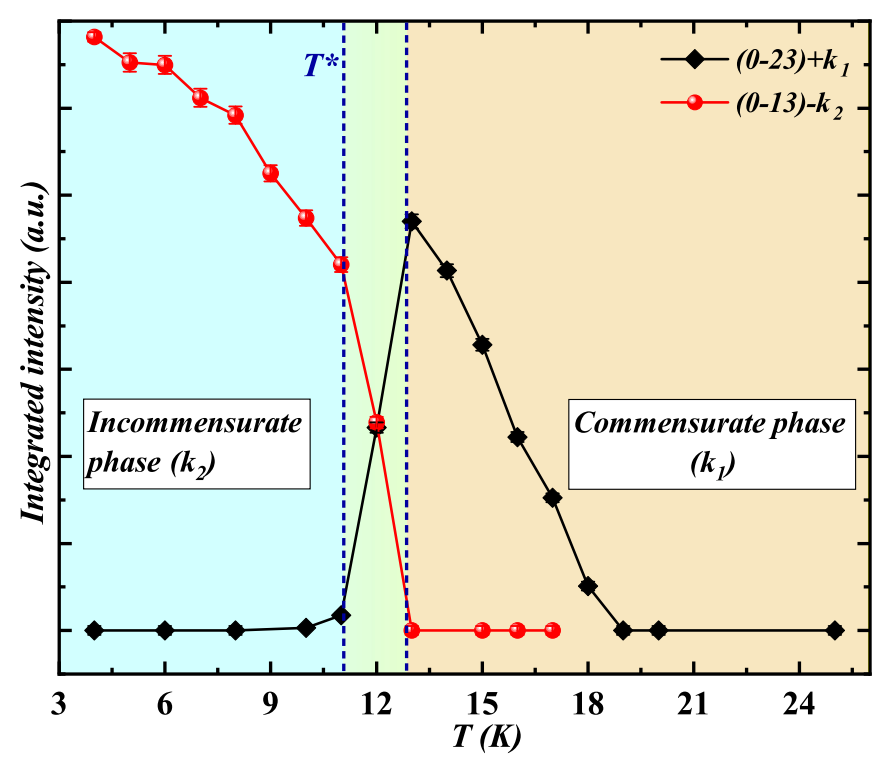

FIG. 6. (a) The main panel shows the temperature dependence of integrated intensity of two magnetic superstructure peaks indicating the thermal evolution of commensurate and incommensurate magnetic phases. There is a narrow region in between these two phases where both propagation vectors $k_{1}$ and $k_{2}$ coexist.

magnetic order at the lowest temperature is incommensurate with the propagation vector $k_{2}=(0.38,0.48,0.5)$. No change in the incommensurate value of $k_{2}$ with temperature has been detected within the measurement accuracy. Moreover, as displayed in Fig. 5(c), the pattern recorded at $T=12 \mathrm{~K}$ shows the presence of magnetic superstructure peaks of both commensurate as well as incommensurate phases.

The two magnetic superstructure peaks observed in the neutron diffraction pattern of $\mathrm{Mn}_{2} \mathrm{~V}_{2} \mathrm{O}_{7}$, namely $(0 \overline{2} 3)^{+}$and $(0 \overline{1} 3)^{-}$satellite peaks for the commensurate and incommensurate phase, respectively, have been recorded at several temperatures. Here, $(h k l)^{ \pm}=(h k l) \pm k_{1} / k_{2}$ denotes a satellite peak of the nuclear $(h k l)$ peak. The integrated intensities of these peaks are plotted as a function of temperature, as shown in Fig. 6. Magnetic intensity fully vanishes above $T_{N 1}=$ $17 \mathrm{~K}$, in close agreement with other bulk measurements. The intensity of the commensurate peak disappears below $T_{N 2}=$ $11.8 \mathrm{~K}$. The presence of magnetic superstructure peaks of both commensurate as well as incommensurate phases in the intermediate narrow region means phase separation takes place indicating a first-order transition from the low-temperature incommensurate to commensurate intermediate temperature phase. This is consistent with the first-order nature of this transition observed from heat capacity and magnetization measurements.

To reveal the magnetic structure, refinement of two data sets collected at 4 and $13 \mathrm{~K}$ has been performed with JANA2006. Magnetic representation analysis sets symmetry restrictions only on the high-temperature commensurate phase. The restrictions imply that the magnetic structure involves either ferromagnetic $\left(\Gamma_{1}\right)$ or antiferromagnetic $\left(\Gamma_{2}\right)$ coupling of the spins related by inversion in the $2 \mathrm{i}$ Wyckoff position. There are no restrictions on the moment direction, and $\mathrm{Mn}$ in the symmetry-independent positions can adopt different sizes and orientations. The refinement, however, was

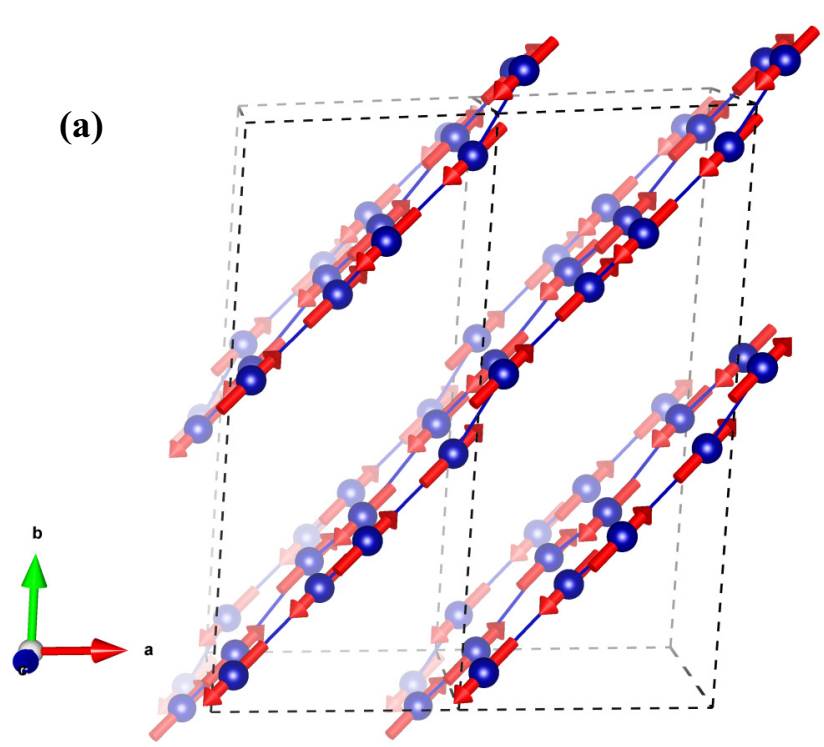

(b)

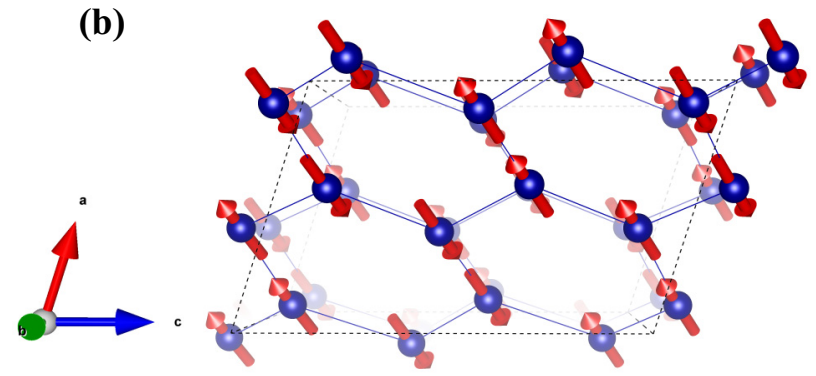

FIG. 7. (a) Perspective view of the spin configuration in the commensurate phase $(T=13 \mathrm{~K})$ is shown. Part (b) shows the arrangement of $\mathrm{Mn}^{2+}$ spins on the distorted honeycomb layer for the same temperature.

constrained to keep the structure collinear with the same ordered moment for all four Mn sites. The obtained solution is shown in Fig. 7, which corresponds to the $\Gamma_{2}$ irreducible representation of the $P \overline{1}$ space group. Magnetic moments have components along all three crystallographic directions: $a, b$, and $c$ axes. The size of the ordered moment at $T=13 \mathrm{~K}$ has been determined to be $2.83 \mu_{B}$ per $\mathrm{Mn}$, where $M_{a}=$ $2.06 \mu_{B}, M_{b}=1.78 \mu_{B}$, and $M_{c}=0.77 \mu_{B}$. In the incommensurate phase, which onsets below $T_{N 2}=11.8 \mathrm{~K}$, symmetry sets no restrictions and we tried to fit the low-temperature diffraction data considering three different models: (a) amplitude modulated structure derived from helical structure, (b) cycloidal structure where moments are rotating with respect to a plane, and (c) proper helical structure. The statistically best fit is obtained for the cycloidal model with equal amplitude of magnetic moment on each $\mathrm{Mn}$ atom. It is yielded for the ordered magnetic moment of $\approx 5.19 \mu_{B}$ per Mn. Figures 8 (a) and 8 (b) show the proposed cycloidal magnetic structure in the low-temperature incommensurate phase from the $c$ axis and the honeycomb plane, respectively. The rotation of the spins is clearly visible in these two projections, while the projection from the honeycomb plane shows the rotation of the moment about the honeycomb plane. The refinement of the data based on models (a)-(c) is presented in Fig. S3 of the Supplemental Material [26]. 
(a)

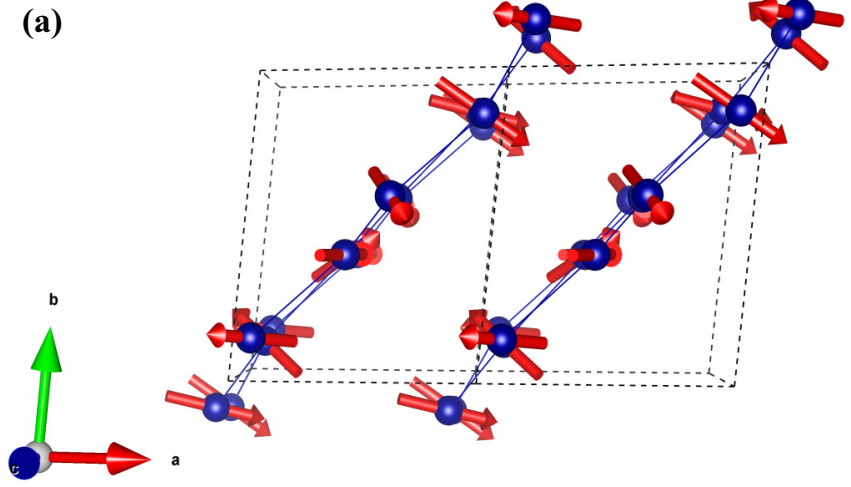

(b)

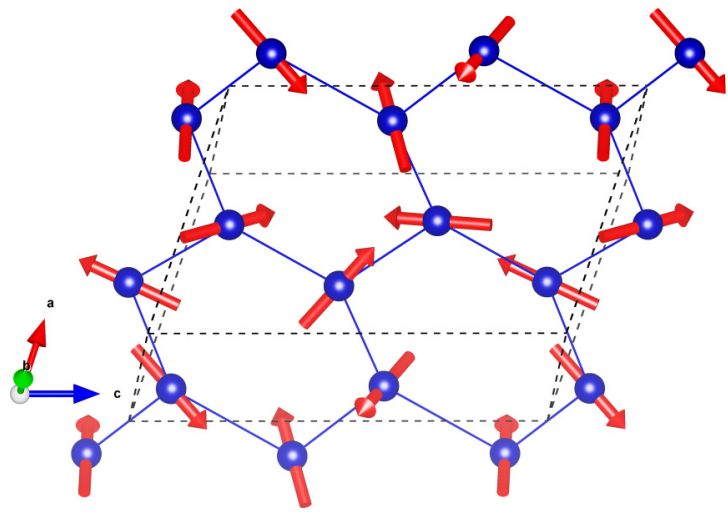

FIG. 8. (a) The perspective view of the spin configuration of $\mathrm{Mn}^{2+}$ in the unit cell is shown for the incommensurate phase below $T_{N 2}=11.8 \mathrm{~K}$. Part (b) depicts a schematic view of the spin configuration in the low- $T$ incommensurate phase obtained from the refinement of $4 \mathrm{~K}$ data.

\section{DISCUSSION}

$\alpha-\mathrm{Mn}_{2} \mathrm{~V}_{2} \mathrm{O}_{7}$ reveals two successive magnetic transitions with two distinct magnetically ordered states, $T_{N 1} \approx 17 \mathrm{~K}$ and $T_{N 2} \approx 11.8 \mathrm{~K}$, from susceptibility as well as heat capacity measurements. Here, $\mathrm{Mn}^{2+}$ ions have a high-spin state, and the system exhibits magnetic anisotropy due to admixture of the orbital moment. Actually, $T_{N 1}$ of $\approx 17 \mathrm{~K}$ is rather low compared to the Curie-Weiss constant $\theta_{C}=-34 \mathrm{~K}$, which indicates the presence of some frustration in the system, which might be responsible for suppressing the 3D magnetic ordering. The second anomaly at $T_{N 2}$ was not reported previously, and it is interesting to find out the nature of this transition. At first, the indication of another magnetic phase was observed from neutron powder diffraction study (although no anomaly was found at this $T_{N 2}$ in the magnetization), but it was nontrivial to determine the magnetic structure solely based on powder data because of the very low symmetry of $\alpha-\mathrm{Mn}_{2} \mathrm{~V}_{2} \mathrm{O}_{7}$ with four different $\mathrm{Mn}$ sites. Hence to solve these two magnetic phases, a single crystal has been prepared, and from our careful neutron diffraction study on the single-crystalline sample of $\mathrm{Mn}_{2} \mathrm{~V}_{2} \mathrm{O}_{7}$ we found commensurate magnetic structure in the intermediate state below $T_{N 1}$ and incommensurate cycloidal-type structure at low temperature.

Microscopically, $\alpha-\mathrm{Mn}_{2} \mathrm{~V}_{2} \mathrm{O}_{7}$ can be viewed as quasi-2D, with the distorted honeycomb layer consisting of $\mathrm{Mn}^{2+}$ ions parallel to the (110) plane, which is the plane along the body diagonal of the unit cell. But the absence of diffuse scattering in the neutron diffraction data indicates strong interaction between the honeycomb layers. Neutron diffraction experiments have shown that this compound adopts a collinear commensurate magnetic structure below $T_{N 1}$ followed by an incommensurate structure with cycloidal modulation of $\mathrm{Mn}$ spins at low temperature, and both phases can be described by the same type of irreducible representation. The incommensurate structure has a predominant antiferromagnetic component, giving rise to satellite peaks in the vicinity of the fundamental AFM Bragg reflection. Now, the existence of this type of commensurate to incommensurate phase transition in insulators is either due to (i) competing exchange interactions, or (ii) relativistic effects such as spin-orbit coupling. While the first one can be found accidentally, the second mechanism depends on lattice symmetry [31,32]. In the case of $\alpha-\mathrm{Mn}_{2} \mathrm{~V}_{2} \mathrm{O}_{7}$, relativistic effects can be discarded as no signature was observed from our experimental data indicating the presence of strong spin-orbit coupling. Considering that spin-orbital coupling will be weaker in $3 d$ transition metals, competing exchange interactions appear to be the main driving mechanism of the incommensurate to commensurate phase transition in our case, which can occur due to anisotropies in the spin Hamiltonian and/or frustration in the lattice [33].

The reduced magnetic moment in the commensurate phase $\left(\approx 2.83 \mu_{B}\right)$ can be taken as a hallmark of quantum fluctuations triggered by the frustrated couplings of Mn spins while the moment increases rapidly below $T_{N 2}\left(\approx 5.19 \mu_{B}\right)$. Now, frustration in the crystal lattice is expected because of very low symmetry and four nonequivalent crystal sites of $\mathrm{Mn}$ where all positions are general. The nature of $\mathrm{Mn}-\mathrm{Mn}$ interaction is superexchange via $\mathrm{O}$ atoms. Because of the distorted nature of Mn-honeycomb layers, there exist different types of Mn-Mn bond lengths with a variable O-environment. Hence, the $\mathrm{Mn}$ $\mathrm{Mn}$ coupling is random on a honeycomb and is supposed to create competing interaction. Furthermore, the period of the spin modulation does not change with $T$ in the incommensurate regime, which indicates some frozen magnetic phase below $T_{N 2}$. Theoretical predictions suggest that frozen spin modulation is possible in a $2 \mathrm{D}$ system with random distribution of coupling [34]. The studied compound is quasi-2D and the theoretical predictions are also in line with our hypothesis.

\section{CONCLUSIONS}

$\alpha-\mathrm{Mn}_{2} \mathrm{~V}_{2} \mathrm{O}_{7}$ reveals two successive magnetic transitions at $T_{N 1}=17 \mathrm{~K}$ and $T_{N 2}=11.8 \mathrm{~K}$ indicating two distinct magnetically ordered phases. The single-crystalline sample has been grown in the floating zone furnace. No further structural changes are observed between 280 and $2 \mathrm{~K}$ in the $\alpha-\mathrm{Mn}_{2} \mathrm{~V}_{2} \mathrm{O}_{7}$ phase. By using a single-crystal neutron diffraction study, we have shown that in the $T$ range $11.8 \leqslant$ $T \leqslant 17 \mathrm{~K}$ the magnetic structure is commensurate with the propagation vector $k_{1}=(0,0.5,0)$, while upon lowering the temperature below $T_{N 2}=11.8 \mathrm{~K}$ a second magnetic phase toward an incommensurate $\left[k_{2}=(0.38,0.48,0.5)\right] 3 \mathrm{D}$ magnetic order emerges. The incommensurate modulation of the $\mathrm{Mn}^{2+}$ spins of the low- $T$ phase is driven by the presence of competing exchange interactions of the next-nearest-neighbor 
$\mathrm{Mn}^{2+}$ spins. The magnetic model at low temperature can be represented by cycloidal modulation along the $a c$ plane, while in the intermediate commensurate phase a collinear arrangement is observed. Our study will stimulate theoretical interest and microscopic studies on the nature and origin of the incommensurate to commensurate phase transition observed in frustrated insulator systems.

Neutron data were taken on the SXD diffractometer at the ISIS Neutron and Muon Source. Information on the data can be accessed through STFC ISIS Facility [35].

\section{ACKNOWLEDGMENTS}

J.S. would like to thank the European Union's Horizon 2020 research and innovation programme under the Marie Skłodowska-Curie Grant Agreement (GA) No. 665593 awarded to the Science and Technology Facilities Council. V.P. would like to acknowledge the Czech Science Foundation (Project No. 18-10504S) for developing the JANA software. We thank G. Stenning for help on magnetization and heat capacity measurements and ISIS Facility for providing beam time on SXD (RB1810466).
[1] Z. Y. Meng, T. C. Lang, S. Wessel, F. F. Assaad, and A. Muramatsu, Nature (London) 464, 847 (2010).

[2] N. Rogado, Q. Huang, J. W. Lynn, A. P. Ramirez, D. Huse, and R. J. Cava, Phys. Rev. B 65, 144443 (2002).

[3] S. Nakatsuji, K. Kuga, K. Kimura, R. Satake, N. Katayama, E. Nishibori, H. Sawa, R. Ishii, M. Hagiwara, and F. Bridges, Science 336, 559 (2012).

[4] F. Ye, S. Chi, H. Cao, B. C. Chakoumakos, J. A. FernandezBaca, R. Custelcean, T. F. Qi, O. B. Korneta, and G. Cao, Phys. Rev. B 85, 180403(R) (2012).

[5] S. Wenzel, L. Bogacz, and W. Janke, Phys. Rev. Lett. 101, 127202 (2008).

[6] U. Tutsch, B. Wolf, S. Wessel, L. Postulka, Y. Tsui, H. O. Jeschke, I. Opahle, T. Saha-Dasgupta, R. Valenti, A. Bruhl, K. Removic-Langer, T. Kretz, H.-W. Lerner, M. Wagner, and M. Lang, Nat. Commun. 5, 5169 (2014).

[7] S. Yan, D. A. Huse, and S. R. White, Science 332, 1173 (2011).

[8] J. A. Sears, M. Songvilay, K. W. Plumb, J. P. Clancy, Y. Qiu, Y. Zhao, D. Parshall, and Y.-J. Kim, Phys. Rev. B 91, 144420 (2015).

[9] J. Wen, S.-L. Yu, S. Li, W. Yu, and J.-X. Li, npj Quantum Mater. 4, 12 (2019).

[10] S. J. Youn, M. H. Fischer, S. H. Rhim, M. Sigrist, and D. F. Agterberg, Phys. Rev. B 85, 220505(R) (2012).

[11] M. Touaiher, K. Rissouli, K. Benkhouja, M. Taibi, J. Aride, A. Boukhari, and B. Heulin, Mater. Chem. Phys. 85, 41 (2004).

[12] Z. He, J.-I. Yamaura, Y. Ueda, and W. Cheng, Phys. Rev. B 79, 092404 (2009).

[13] A. A. Tsirlin, O. Janson, and H. Rosner, Phys. Rev. B 82, 144416 (2010).

[14] Z. He and Y. Ueda, Phys. Rev. B 77, 052402 (2008).

[15] Z. He, J.-I. Yamaura, Y. Ueda, and W. Cheng, J. Solid State Chem. 182, 2526 (2009).

[16] C. Calvo and R. Faggiani, Acta Crystallogr. B 31, 603 (1975).
[17] J. Sannigrahi, S. Bhowal, S. Giri, S. Majumdar, and I. Dasgupta, Phys. Rev. B 91, 220407(R) (2015).

[18] J. Sannigrahi, S. Giri, and S. Majumdar, Solid State Commun. 228, 10 (2016).

[19] J. Sannigrahi, S. Giri, and S. Majumdar, J. Phys. Chem. Solids 101, 1 (2017).

[20] Y. C. Sun, Z. W. Ouyang, Y. Xiao, Y. Su, E. Feng, Z. Fu, W. T. Jin, M. Zbiri, Z. C. Xia, J. F. Wang, and G. H. Rao, Mater. Res. Express 4, 046101 (2017).

[21] J. H. Liao, F. Leroux, C. Payen, D. Guyomard, and Y. Piffard, J. Solid State Chem. 121, 214 (1996).

[22] Z. He, Y. Ueda, and M. Itoh, Solid State Commun. 147, 138 (2008).

[23] Z. He and Y. Ueda, J. Cryst. Growth 310, 171 (2008).

[24] Z. Chuan-Cang, L. Fa-Min, and D. Peng, Chin. Phys. B 18, 5055 (2009).

[25] A. A. Fotiev and L. L. Surat, Russ. J. Inorg. Chem. 27, 4 (1982).

[26] See Supplemental Material at http://link.aps.org/supplemental/ 10.1103/PhysRevMaterials.3.113401 for details on the refinement.

[27] Z. He and Y. Ueda, J. Solid State Chem. 181, 235 (2008).

[28] D. A. Keen, M. J. Gutmann, and C. C. Wilson, J. Appl. Crystallogr. 39, 714 (2006).

[29] M. Gutmann, Acta Crystallogr. Sect. A 61, c164 (2005).

[30] V. Petříček, M. Dušek, and L. Palatinus, Crystallographic computing system JANA2006: General features, Z. Kristallogr. 229, 345 (2014).

[31] B. Roessli, J. Schefer, G. A. Petrakovskii, B. Ouladdiaf, M. Boehm, U. Staub, A. Vorotinov, and L. Bezmaternikh, Phys. Rev. Lett. 86, 1885 (2001).

[32] D. Vaknin, J. L. Zarestky, J.-P. Rivera, and H. Schmid, Phys. Rev. Lett. 92, 207201 (2004).

[33] P. Bak, Rep. Prog. Phys. 45, 587 (1982).

[34] E. C. Marino, Phys. Rev. B 65, 054418 (2002).

[35] J. Sannigrahi et al. (2018), https://doi.org/10.5286/ISIS.E. RB1810466. 